\title{
Is periodontal disease associated with poor pregnancy outcomes?
}

\author{
What is the strength of association between periodontal disease in mothers \\ and birth of their infants preterm or with low birthweights?
}

\section{Vergnes J-N, Sixou M.}

Preterm low birth weight and maternal periodontal status: a metaanalysis. Am J Obstet Gynecol 2007; 196:135.e1-135.e7

Data sources Searches for studies were made using Medline, Embase, LILACS, Biosis and PASCAL and the reference lists of all identified articles were also checked for relevant reports. There were no restrictions on language of publication.

Study selection Studies were selected if they were of cohort, case-control or cross-sectional design, with exposure defined as mother's periodontal disease and cases being defined as delivery at $<37$ weeks' gestation or infant with a birthweight $<2500 \mathrm{~g}$. Two reviewers independently evaluated articles for eligibility.

Data extraction and synthesis Data from all eligible studies were extracted and summarised independently by two reviewers. Studies were combined to estimate the pooled odds ratio (OR) with $95 \%$ confidence intervals $(\mathrm{Cl})$ using the inverse variance method. Both fixed-effects and random effects models (based on the DerSimonian-Laird method) were used to calculate the overall OR.

Results Seventeen articles met the inclusion criteria. A total of 7151 women participated in the studies, 1056 of whom delivered a preterm and/ or low birthweight infant. The overall OR was $2.83(95 \% \mathrm{Cl}$, $1.95-4.10 ; \mathrm{P} 0.0001)$. This pooled value required cautious interpretation because there appeared to be a clear trend for the better quality studies to produce a lower strength of association.

Conclusions These findings indicate a possible association, but this needs to be confirmed by large, well-designed, multicentre trials.

Address for correspondence: Jean-Noël Vergnes, Dental School,

Department of Epidemiology, 3, Chemin des Maraichers, 31400 Toulouse, France. E-mail: Sixou@cict.fr

\section{Commentary}

Conflicting findings regarding the association between poor pregnancy outcomes and periodontal disease flourish in the literature: most studies report a positive association which, if real and independent, would provide new tools to reduce the burden of many neonatal outcomes. ${ }^{1}$ Unfortunately, it remains unclear the extent to which bias and confounding have affected the reports, and a systematic appraisal of the quality and strength of the evidence is missing.

In this article, the authors aimed, "to assess the effect of maternal periodontal disease on preterm delivery and/ or birth of low weight infants" using a systematic review of the observational evidence available. The aim can indeed be achieved by the systematic evaluation of the quality of the studies conducted; subgroup analyses to unravel the impact of bias and confounding; and special consideration of the evidence provided by the best studies.

The authors combined case-control, cohort and cross-sectional studies, ignoring the fact that pooling results taken from several observational studies and different study designs is likely to produce "very precise but equally spurious results" because of the lack of comparability of study groups: these analyses combine average estimates from studies designed to answer different questions in different populations. ${ }^{2}$ The author also state that the MOOSE guidelines (for metaanalyses and systematic reviews of observational studies in epidemiology $)^{3}$ were followed, but this is only partially correct.

The main goal of meta-analyses of observational studies is not to provide pooled estimates of association for the assessment of the strength of the evidence, but to provide a tool for the assessment of the heterogeneity and the quality of the available evidence. When observational studies are pooled in a meta-analysis, different type of studies originating from different study populations are merged into a single study group and information about the comparability of different subsets of study participants is lost. For example, any meta-analysis of case-control studies violates the assumption that cases and controls originated from the same underlying population or, in other words, that controls represent a random sample from the whole population giving rise to the cases. ${ }^{4}$

The study confirms that insignificant-to strong associations between periodontal disease and poor pregnancy outcomes have been reported and that, interestingly, the strength of the association depends of the quality of the studies. The best-ranking studies ${ }^{5,6}$ fail to report a significant association. The attempts to weight pooled results using quality scores are not enough to remove the noise.

The cross-sectional and case-control studies included were rather small, and most of the results were not significant. In only two cohort studies, ${ }^{7,8}$ which actually had the lowest quality scores in the review, did the authors reported a significant association, whereas the best and largest study included in the review (number of subjects, 3738) reported no association. ${ }^{6}$ 
The authors conclude that the findings "indicate a likely association", but that this needs to be confirmed by large, well-designed, multicentre trials. The negative results of the best and largest study $^{6}$ included in this review and a recently published, multicentre, randomised controlled trial ${ }^{9}$ suggest that the query has been answered.

\section{Practice point}

Even though most available studies report a positive association between periodontal disease and poor pregnancy outcomes, the evidence provided by the best studies counteracts this and suggests that bias and confounding may account for a considerable proportion of the association.

Rodrigo López

Department of Community Oral Health and Paediatric Dentistry, Faculty of Health Sciences, University of Aarhus, Aarhus, Denmark
1. Petrou S, Sach T, Davidson L. The long-term costs of preterm birth and low birth weight: results of a systematic review. Child Care Health Dev 2001; 27:97-115.

2. Lopez R. Periodontal disease, preterm birth and low birthweight. Does periodontal disease in pregnant mothers increase the risk of preterm birth or low birthweight? Evid Based Dent 2005; 6:90-91.

3. Stroup DF, Berlin JA, Morton SC et al. Meta-analysis of observational studies in epidemiology: a proposal for reporting. Meta-analysis of observational studies in epidemiology (MOOSE) group. J Am Med Assoc 2000; 283:2008-2012.

4. Rothman KJ, Greenland S Modern Epidemiology. 2nd edn, Lippincott-Raven, U.S.A., 1998 93-114.

5. Lunardelli AN, Peres MA. Is there an association between periodontal disease, prematurity and low birth weight? A population-based study. J Clin Periodontol 2005; 32:938-946.

6. Moore S, Moore S, Ide M, Coward PY et al. A prospective study to investigate the relationship between periodontal disease and adverse pregnancy outcome. Br Dent J 2004; 197:251-258.

7. Jeffcoat MK, Hauth JC, Geurs NC et al. Periodontal infection and preterm birth. Results of a prospective study. J Am Dent Assoc 2001; 132:875-879.

8. Dortbudak O, Eberhardt R, Ulm M, Persson GR.. Periodontitis, a marker of risk in pregnancy for preterm birth. J Clin Periodontol 2005; 32:45-52.

9. Michalowicz BS, Hodges JS, DiAngelis AJ et al. Treatment of periodontal disease and the risk of preterm birth. N Engl J Med 2006; 355:1885-1894.

Evidence-Based Dentistry (2007) 8, 114-115. doi:10.1038/sj.ebd.6400531 